Pacific Journal of Mathematic 


\title{
ITERATES OF ARITHMETIC FUNCTIONS AND A PROPERTY OF THE SEQUENCE OF PRIMES
}

\author{
HaR OLd N. ShaPIRo
}

1. Introduction. In a previous paper [2], the author has investigated certain properties of the iterates of arithmetic functions which are of the following form. For $n=\prod p_{i}^{a_{1}}$,

$$
f(n)=\prod f\left(p_{i}\right)\left[A\left(p_{i}\right)\right]^{a_{i}-1},
$$

where $f\left(p_{i}\right)$ is an integer, $1<f\left(p_{i}\right)<p_{i}$, and $A\left(p_{i}\right)$ is an integer $\leq p_{i}$, for odd primes $p_{i}$; whereas $f(2)=1, A(2)=2$. We shall denote the set of these arithmetic functions by $K$. These conditions ensure that for $n>2, f(n)<n$, and hence if $f^{k}(n)$ denotes the $k$-th iterate of $f$ there is a unique integer $k$ such that

$$
f^{k}(n)=2 \text {. }
$$

For this $k$ we write $k=C_{f}(n)$. We define

$$
C_{f}(1)=C_{f}(2)=0 .
$$

In this paper we propose to consider the problem of determining a $g \in K$ such that for all odd primes $p$, and all $f \in K$,

$$
C_{g}(p) \geq C_{f}(p) .
$$

The solution to this problem produces an interesting property of the sequence of primes in that we shall show that (1.3) is equivalent to having $g$ skip down through the primes. More precisely, if $p_{1}=2, p_{2}=3, \cdots$, and in general $p_{i}$ denotes the $i$-th prime, (1.3) is equivalent to having $g(3)=2, g(5)=4$ or 3 , and

$$
g\left(p_{i}\right)=p_{i-1} \quad \text { for } i>3 .
$$

2. A theorem concerning functions of $K$. In carrying out the proof of the result

Received September 10, 1952.

Pacific J. Math. 3 (1953), 647-655 
stated in the introduction, we shall require a certain property of the iterates of the functions of $K$, which we now derive.

For $n=\prod p_{i}^{a_{i}}$, we define the arithmetic function $A(n)$ as

$$
A(n)=\prod\left[A\left(p_{i}\right)\right]^{a_{i}}
$$

where the $A\left(p_{i}\right)$ are as given in (1.1). It then follows that, for all integers $m$ and $n$,

$$
A(m n)=A(m) A(n) \text { and } A(n) \leq n
$$

LEмма 2.1. For any divisor $d$ of $n$, we have for $f \in K$,

$$
\frac{A(d) f(n)}{f(d)} \leq n
$$

where $A(d) f(n) / f(d)$ is an integer.

Proof. We can write

$$
\begin{aligned}
f(n) & =A(n) \prod_{p \mid n} \frac{f(p)}{A(p)} \\
& =A(n) \prod_{p \mid d} \frac{f(p)}{A(p)} \prod_{\substack{p \mid n \\
p \nmid d}} \frac{f(p)}{A(p)} \\
& =A(n) \frac{f(d)}{A(d)} \cdot \frac{f\left(d^{\prime}\right)}{A\left(d^{\prime}\right)},
\end{aligned}
$$

where

$$
d^{\prime}=\prod_{\substack{p \mid n \\ p \nmid d}} p
$$

so that $d^{\prime}$ divides $n$. Since $A(n)$ is completely multiplicative, we have then

$$
A(n)=A\left(\frac{n}{d^{\prime}}\right) A\left(d^{\prime}\right), \quad \text { or } \quad \frac{A(n)}{A\left(d^{\prime}\right)}=A\left(\frac{n}{d^{\prime}}\right) \text {. }
$$

Hence

$$
\frac{A(d) f(n)}{f(d)}=A\left(\frac{n}{d^{\prime}}\right) f\left(d^{\prime}\right) \leq n,
$$


where clearly $A(d) f(n) / f(d)$ is an integer.

Lemma 2.2. For $f \in K$, if $e(n)=0$ or 1 according as $n$ is odd or even,

$$
C_{f}(2 n) \leq C_{f}(n)+e(n) .
$$

Proof. Since $f \in K$, we have $f(2)=1, A(2)=2$, and hence

$$
f(2 n)=2 f(n) \text { or } f(n) \text {, }
$$

where if $n$ is odd $f(2 n)=f(n)$ and $C_{f}(2 n)=C_{f}(n)$. Otherwise, continuing, we have

$$
f^{2}(2 n)=2 f^{2}(n) \text { or } f^{2}(n)
$$

and in general

$$
f^{k}(2 n)=2 f^{k}(n) \text { or } f^{k}(n) .
$$

Then taking $k=C_{f}(n)$ we get

$$
f^{k}(2 n)=4 \text { or } 2,
$$

so that

$$
C_{f}(2 n) \leq k+1=C_{f}(n)+1 .
$$

THЕОR Ем 2.1. If $x$ is such that for all $z<x, C_{f}(z)<C_{f}(x)$, where $f \in K$, then for all $y$,

$$
C_{f}(x y) \leq C_{f}(x)+C_{f}(y)+e(x) .
$$

Proof. We have

$$
f(x y)=\frac{f(x) f(y) A(d)}{f(d)}
$$

where $d=(x, y)$. Letting

$$
\beta_{1}=\frac{f(x) A(d)}{f(d)}
$$

we know from Lemma 2.1 that $\beta_{1}$ is an integer less than or equal to $x$; and

$$
f(x y)=\beta_{1} f(y) .
$$


Then similarly

$$
f^{2}(x y)=\beta_{2} f^{2}(y),
$$

where

$$
\begin{aligned}
& \beta_{2}=\frac{f\left(\beta_{1}\right) A(\gamma)}{f(\gamma)} \leq \beta_{1} \leq x, \\
& \gamma=\left(\beta_{1}, f(y)\right) .
\end{aligned}
$$

Thus in general we have:

$$
f^{k}(x y)=\beta_{k} f^{k}(y), \quad \beta_{k} \leq \beta_{k-1} \leq \cdots \leq \beta_{1} \leq x,
$$

so that, letting $k=C_{f}(y)$, we get

$$
f^{k}(x y)=2 \beta_{k}, \quad \beta_{k} \leq x .
$$

Then if $\beta_{k}<x$ we have via Lemma 2.2, and our hypothesis,

$$
\begin{aligned}
C_{f}(x y) & =C_{f}(y)+C_{f}\left(2 \beta_{k}\right) \\
& \leq C_{f}(y)+C_{f}\left(\beta_{k}\right)+1 \\
& \leq C_{f}(y)+C_{f}(x) .
\end{aligned}
$$

On the other hand, if $\beta_{k}=x$ we have

$$
\begin{aligned}
C_{f}(x y) & =C_{f}(y)+C_{f}(2 x) \\
& \leq C_{f}(y)+C_{f}(x)+e(x),
\end{aligned}
$$

and the theorem is proved.

3. Derivation of the main result. In carrying out the proof of the equivalence of (1.3) and (1.4) we shall need certain estimates from elementary prime number theory. These results are given in the following lemma. As is conventional, we shall write $p_{1}=2, p_{2}=3, \ldots$, and let $p_{i}$ denote the $i$-th prime.

Lемма 3.1. Letting $\pi(x)=$ the number of primes $\leq x$, we have

(a) $2 p_{i-2}>p_{i}$ for $i>5$,

(b) for all positive integers $x>2$,

$$
\pi(x)-\pi\left(\frac{x}{7}\right)>\sqrt{x}
$$


Proof. Both of the above are deducible from a result of Ramanujan [1] which asserts that for $x>300$,

$$
\pi(x)-\pi\left(\frac{x}{2}\right)>\frac{x / 6-3 \sqrt{x}}{\log x}
$$

Ramanujan gives explicitly the result that for $x \geq 11$,

$$
\pi(x)-\pi\left(\frac{x}{2}\right) \geq 2 \text {, }
$$

which implies (a). As for (b), we note that since, for $x \geq 10,590$,

$$
\frac{1}{\log x}\left(\frac{1}{6} x-3 \sqrt{x}\right)>\sqrt{x}
$$

we have (3.1) for all $x>10,590$. We can check (3.1) for all $x<10,590$ very quickly. We check up to $x=17$. Then let

$$
\begin{array}{lll}
a_{0}=10,590, & a_{1}=2,309, & a_{2}=653, \\
a_{3}=229, & a_{4}=103, & a_{5}=59, \\
a_{6}=37, & a_{7}=23, & a_{8}=17 ;
\end{array}
$$

inspecting tables of primes, we see that these numbers have the property that

$$
\pi\left(a_{i+1}\right)-\pi\left(\frac{a_{i}}{7}\right)>\sqrt{a_{i}},
$$

which completes the proof of (b).

We now give our main result as:

THEOREм 3.1. If $g(x), g \in K$, is such that $C_{g}(x)$ is maximal for all primes $p$, that is $C_{g}(p) \geq C_{f}(p)$ for all $f \in K$ and all $p$, then $g(3)=2, g(5)=4$ or 3 , and, for $i>3, g\left(p_{i}\right)=p_{i-1}$.

Proof. Since $g \in K$, we clearly have $g(2)=g(1)=1$; and $g(3)=2$. Now in choosing $g(5)<5$, we consider all possible values and choose the one which makes $C_{g}(5)$ a maximum. Symbolically, we may write

$$
g(5)=C^{-1}\{\max [C(j), 0<j<5]\}=4 \text { or } 3 .
$$

Thus $g(5)$ has two possible values 4 or 3 . Similarly proceeding to $p_{4}=7$ and $p_{5}=11$ we have 


$$
g(7)=C^{-1}\{\max [C(j), 0<j<7]\}=5
$$

and

$$
g(11)=C^{-1}\{\max [C(j), 0<j<11]\}=7 .
$$

In general, for the $i$-th prime we must have

$$
g\left(p_{i}\right)=C^{-1}\left\{\max \left[C(j), 0<j<p_{i}\right]\right\} .
$$

Now it would seem that the determination of this value $g\left(p_{i}\right)$, since it depends upon the $C(j)$, which in turn may require the values of $g(n)$ for composite $n$, would remain undetermined so long as nothing is said about the function $A(n)$. However, as we shall see, the maximum of these $C(j)$, required in (3.3), will turn out to be completely independent of $A(n)$.

We have noted that the theorem is true for $i=4,5$. Proceeding by induction, assume it true for all $i^{\prime}, 4 \leq i^{\prime}<n$, and consider $n>5$. From (3.3) we see that in order to complete the proof we need only show that for any $x$ such that

$$
p_{n}>x>p_{n-1}
$$

we must have

$$
C(x)<C\left(p_{n-1}\right)=n-2 .
$$

Assume that for some $x$ satisfying $(3.4),(3.5)$ is false, and let $x$ be the smallest one satisfying (3.4) for which

$$
C(x) \geq n-2 \text {. }
$$

Then we have also

$$
C(g(x)) \geq n-3 .
$$

We shall now show that $g(x) \neq p_{n-1}$. For suppose that $g(x)=p_{n-1}$. Then $x$. must have a prime divisor $q$ such that $g(q)=p_{n-1}$. But from (3.4) we see that $q \leq p_{n-1}$, which is impossible.

If $g(x)<p_{n-1}$, by our inductive hypothesis we would have

$$
C(g(x)) \leq C\left(p_{n-2}\right)=n-3 \text {. }
$$

Now if $C(g(x))=n-3$, it would follow that $g(x)=p_{n-1}$. This in turn implies that $p_{n-1}$ divides $x$. Since $x \neq p_{n-1}$, this yields 


$$
x \geq 2 p_{n-1}>p_{n}
$$

which is a contradiction. The only alternative left is that $C(g(x))<n-3$, which contradicts (3.7). Thus we conclude that $g(x)>p_{n-1}$ so that we must have

$$
p_{n}>x>g(x)>p_{n-1}
$$

Since $x$ is the smallest integer satisfying (3.4) and not (3.5), we must have $C(g(x))<n-2$ or $C(x) \leq n-2$; hence

$$
C(x)=n-2
$$

Now $x$ is not even, for if it were we would have

$$
g(x) \leq \frac{x}{2}<\frac{p_{n}}{2}<p_{n-1}
$$

which is a contradiction. Also $x$ is not divisible by 3 for $n>5$; for if it were, $g(x)$ would be even and we would get, using Lemma 3.1 (a),

$$
g^{2}(x) \leq \frac{g(x)}{2}<\frac{p_{n}}{2}<p_{n-2}
$$

But then

$$
C\left(g^{2}(x)\right) \leq C\left(p_{n-3}\right)=n-4
$$

If the inequality sign holds, this implies $C(x)<n-2$ in contradiction to (3.10). On the other hand, if the equality sign holds then $g^{2}(x)=p_{n-3}$. This in turn implies that $p_{n-2}$ divides $g(x)$. If $g(x) \neq p_{n-2}$, then

$$
g(x) \geq 2 p_{n-2}>p_{n}
$$

which is impossible. Finally, $g(x)=p_{n-2}$ implies that $x$ is divisible by $p_{n-1}$, which is impossible.

Also, if $x$ is not divisible by 5 , the argument is the same as for 3 . On the other hand if $g(5)=3$, and $x$ is divisible by 5 , it results that $g^{2}(5)$ is even, and hence

$$
g^{3}(x) \leq \frac{1}{2} g^{2}(x) \leq \frac{1}{2} \cdot \frac{2}{3} g(x) \leq \frac{1}{2} \cdot \frac{2}{3} \cdot \frac{3}{5} x \leq \frac{p_{n}}{5}<p_{n-4} \cdot
$$

But this again implies that $C(x)<n-2$, which is impossible. 
Suppose then that $p \geq 7$ is the smallest prime which divides $x$. Since $x$ is composite, $1<x / p<x$ and $p \leq \sqrt{x}$. It is clear from (3.3) and our inductive hypothesis that for $z<p, C(z)<C(p)$. Hence via Theorem 2.1 we have

$$
n-2=C(x) \leq C\left(\frac{x}{p}\right)+C(p) .
$$

Via our inductive hypothesis we see that, since $p \leq \sqrt{x}$,

$$
C(p) \leq \pi(\sqrt{x})
$$

so that

$$
C\left(\frac{x}{p}\right)+\pi(\sqrt{x}) \geq n-2 .
$$

Since

$$
x>p_{n-1}>\frac{x}{7} \geq \frac{x}{p},
$$

and

$$
C(x)=C\left(p_{n-1}\right)=n-2,
$$

we have

$$
C(x)-C\left(\frac{x}{p}\right) \geq \pi(x)-\pi\left(\frac{x}{7}\right)>\sqrt{x},
$$

by Lemma 3.1 (b); and

$$
C\left(\frac{x}{p}\right)<n-2-\sqrt{x}
$$

Combining (3.11) and (3.12) yields $\pi(\sqrt{x})>\sqrt{x}$, an obvious contradiction: thus the proof of the theorem is completed.

4. Some remarks and generalizations. From the above we note that imposing the condition that the function $C_{f}(n)$ be maximal at the primes determines uniquely the values of $f(n)$ at the primes without restricting $A(n)$ in any way. This is natural from a certain point of view, since the function $A(n)$ plays a role only in evaluating $f(n)$ for powers of a prime. This might lead one to suspect that requiring that $C_{f}(n)$ be maximal at the $p_{i}^{2}$ in addition to the $p_{i}$ would also 
determine the values of $A(n)$. This is in fact the case, and one may prove (we omit the proof since it is long and very similar to that of $\$ 3$ ):

THEOREM 4.1. If $C_{g}(x)$ is maximal at the primes and squares of primes, then $A_{g}(3)=2$ or $3, A_{g}(5)=5$ or 4 , and for $p_{i}>5, A_{g}\left(p_{i}\right)=p_{i}$ or $p_{i-1}$. Furthermore this same maximal $C_{g}(x)$ is realized for any admis sible choice of the $A_{g}\left(p_{i}\right)$ (that is, as either $p_{i}$ or $p_{i-1}$ ).

\section{REFERENCES}

1. S. Ramanujan, Proof of Bertrand's postulate, J. Indian Math. Soc. 11 (1919), xxx-xxx.

2. Harold N. Shapiro, On the iterates of a certain class of arithmetic functions, Comm. Pure Appl. Math. 3 (1950), 259-272.

New York University 



\section{PACIFIC JOURNAL OF MATHEMATICS}

\section{EDITORS}

\author{
R. M. BOEINSON \\ University of California \\ Berkeley 4, California \\ E. HewitT \\ University of Washington \\ Seattle 5 , Washington
}

\section{R. P. DILWOR TH}

California Institute of Technology

Pasadena 4, California

E. F. BECKENBACH

University of California

Los Angeles 24, California

\section{ASSOCIATE EDITORS}

$\begin{array}{ll}\text { H. BUSEMANN } & \text { P. R. HALMOS } \\ \text { HERBERT FFDERER } & \text { IIEINZ HOPF } \\ \text { MARSHALL IJALL } & \text { R. D. JAMES }\end{array}$

\author{
BøRGE JESSEN \\ PAUL LÉVY \\ GEORGE PÓLYA
}

\author{
J. J. STOKER \\ E. G. STR AUS \\ KÔSAKU YOSIDA
}

\section{SFONSORS}

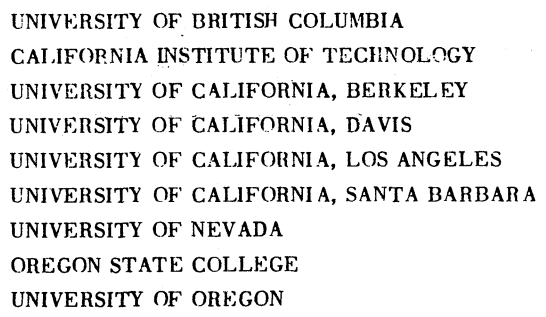

\author{
UNIVERSITY OF SOUTHERN CALIFORNIA \\ STANFORD RESEARCH INSTITUTE \\ STANFORD UNIVERSITY \\ WASHINGTON STATE COLLEGE \\ UNIVERSITY OF WASHINGTON \\ AMERICAN MATHEMATICAL SOCIETY \\ NATIONAL BUREAU OF STANDARDS, \\ INSTITUTE FOR NUMERICAL ANALYSIS
}

Mathematical papers intended for publication in the Pacific Journal of Mathematics should be typewritten (double spaced), and the author should keep a complete copy. Manuscripts may be sent to any of the editors except Robinson, whose term expires with the completion of the present volume; they might also be sent to M.M. Schiffer, Stanford University, Stanford, California, who is succeeding Robinson. All other communications to the editors should be addressed to the managing editor, E. F. Beckenbach, at the address given above.

Authors are entitled to receive 100 free reprints of their published papers and may obtain additional copies at cost.

The Pacific Journal of Mathematics is published quarterly, in March, June, September, and December. The price per volume (4 numbers) is $\$ 8.00$; single issues, $\$ 2.50$. Special price to individual faculty members of supporting institutions and to individual members of the American Mathematical Society: $\$ 4.00$ per volume; single issues, $\$ 1.25$.

Subscriptions, orders for back numbers, and changes of address should be sent to the publishers, University of California Press, Berkeley 4, California.

Printed at Ann Arbor, Michigan. Entered as second class matter at the Post Office, Berkeley, California.

\section{UNIVERSITY OF CALIFORNIA PRESS • BERKELEY AND LOS ANGELES}




\section{Pacific Journal of Mathematics}

\section{Vol. 3, No. 3 \\ May, 1953}

L. Carlitz, Some theorems on generalized Dedekind sums ............ 513

L. Carlitz, The reciprocity theorem for Dedekind sums ............. 523

Edward Richard Fadell, Identifications in singular homology theory..... . . 529

Harley M. Flanders, A method of general linear frames in Riemannian

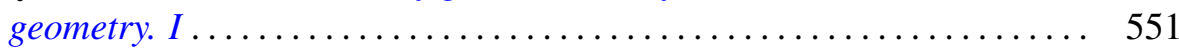

Watson Bryan Fulks, The Neumann problem for the heat equation ........ 567

Paul R. Garabedian, Orthogonal harmonic polynomials.............. 585

R. E. Greenwood and Andrew Mattei Gleason, Distribution of round-off errors for running averages .............................. 605

Arthur Eugene Livingston, The space $H^{p}, 0<p<1$, is not normable ... 613

M. N. Mikhail, On the order of the reciprocal set of a basic set of polynomials .......................................... 617

Louis Joel Mordell, On the linear independence of algebraic numbers . . . . 625

Leo Sario, Alternating method on arbitrary Riemann surfaces .......... 631

Harold Nathaniel Shapiro, Iterates of arithmetic functions and a property of the sequence of primes.............................. 647

H. Shniad, Convexity properties of integral means of analytic functions . . . . 657

Marlow C. Sholander, Plane geometries from convex plates ........... 667 